\title{
METRIC DEFINITION OF THE LINEAR STRUCTURE
}

\author{
RUSSELL G. BILYEU
}

Mazur and Ulam [1] proved that the metric of a real normed linear space determines the linear operations. The following converse of an elementary theorem gives an explicit characterization of convex combinations in terms of the metric $d$ of such a space.

THEOREM. If $0<a<1$ and if $x, y$, and $z$ are members of a real normed linear space such that for each w in the space

$$
d(w, z) \leqq a d(w, x)+(1-a) d(w, y)
$$

then $z=a x+(1-a) y$.

Proof. We may assume $z=0$ and $a \leqq 1 / 2$. Taking $a=1 / 2$, suppose $2 d(w, 0) \leqq d(w, x)+d(w, y)$ for all $w$. Then 0 is in the set $T$ of those nonnegative integers $t$ for which, for each positive integer $m$,

$$
(2 m+t) d(x+y, 0) \leqq d(m x+m y, x)+d(m x+m y, y) .
$$

Choosing $t$ in $T$, observe that if $m$ is a positive integer then so is $2 m$, and

$$
(4 m+t) d(x+y, 0) \leqq d(2 m x+2 m y, x)+d(2 m x+2 m y, y) .
$$

Setting $w=(2 m-1) x+2 m y$ in the hypothesis leads to

$$
d(2 m x+2 m y, x) \leqq d(m x+m y, x)+(m-1 / 2) d(x+y, 0) .
$$

This last statement also holds with $x$ and $y$ interchanged, and from these inequalities we conclude that

$$
\begin{aligned}
(4 m+t) d(x+y, 0) \leqq & d(m x+m y, x)+d(m x+m y, y) \\
& +(2 m-1) d(x+y, 0) .
\end{aligned}
$$

Therefore $(2 m+t+1) d(x+y, 0) \leqq d(m x+m y, x)+d(m x+m y, \quad y)$. By induction, $T$ contains all nonnegative integers. In particular, if $t$ is a nonnegative integer then

$$
(2+t) d(x+y, 0) \leqq d(y, 0)+d(x, 0) .
$$

This implies $x+y=0$, as desired.

Now if $0<a<1 / 2$ and if

Received by the editors September 19, 1969.

AMS Subject Classifications. Primary 4610; Secondary 5250.

Key Words and Phrases. Metric definition, linear structure, normed linear space, convex combinations. 


$$
d(w, 0) \leqq a d(w, x)+(1-a) d(w, y),
$$

then

$$
\begin{aligned}
d(w, 0) & \leqq d(a w, a x)+d(w-a w, y-a y) \\
& \leqq d(a w, a x)+d(a w, y-a y)+d(w-2 a w, 0) \\
& =d(a w, a x)+d(a w, y-a y)+(1-2 a) d(w, 0) .
\end{aligned}
$$

Therefore $2 d(a w, 0) \leqq d(a w, a x)+d(a w, y-a y)$. If these inequalities are assumed to hold for all $w$, then by the previous argument, $a x+y$ $-a y=0$.

\section{BIBLIOGRAPHY}

1. S. Mazur and S. Ulam, Sur les transformations isometriques d'espaces vectoriels, C.R. Acad. Sci. Paris 194 (1932), 946-948.

North Texas State University, Denton, Texas 76203 